\title{
The dynamic molecular landscape of malignant melanomas arising from congenital or common nevi
}

\author{
Olivier Harou $^{1,2}$, Garance Tondeur ${ }^{1,4}$, Françoise Descotes ${ }^{5}$, Brigitte Balme ${ }^{1}$, Lauriane Depaepe ${ }^{1}$, Pierre-Paul Bringuier ${ }^{6}$ Julie Caramel $^{4}$, Luc \\ Thomas $^{2,3,4}$, Stéphane Dalle ${ }^{2,3,4, \#}$ and Jonathan Lopez $z^{2,4,5, \#, *}$ \\ ${ }^{1}$ Department of Pathology, Hospices Civils de Lyon, Centre Hospitalier Lyon-Sud, France \\ ${ }^{2}$ Université de Lyon, Université Claude Bernard Lyon 1, Lyon, France \\ ${ }^{3}$ Dermatology Unit, Hospices Civils de Lyon, Centre Hospitalier Lyon-Sud, France \\ ${ }^{4}$ Cancer Research Center of Lyon Univ Lyon, Université Claude Bernard Lyon 1, INSERM1052, CNRS5286, Centre Léon Bérard, Lyon 69008, France \\ ${ }^{5}$ Department of Biochemistry and Molecular Biology, Hospices Civils de Lyon, Centre Hospitalier Lyon-Sud, France \\ ${ }^{6}$ Department of Pathology, Hospices Civils de Lyon, Groupement Hospitalier Est, France \\ \#These authors share senior authorship
}

\begin{abstract}
Background. Some cutaneous melanomas are arising from nevus, that can be either from the congenital or the common type.

Objective: We aimed at comparing: i) the genomic landscape of small/medium congenital versus common nevi and ii) explore whether there is a different mutational pattern acquired during progression to melanoma between this two groups.

Methods: We analysed BRAF, NRAS and TERT promoter status on micro-dissected melanocytic nevi and performed massively parallel sequencing on paired melanomas in 42 patients, half from the congenital and half from the common type.

Results: Both common and small/medium congenital nevi mainly harboured BRAF V600E mutation at a similar rate. During melanoma progression, both groups acquired recurrent second hit TERT promoter mutations. Additional genomic alterations affecting oncogenic pathways also accumulated during progression, without a specific pattern.
\end{abstract}

Conclusions: Small/medium congenital and common nevi shared the same mutational landscape and did not show any difference during progression to melanoma.

\section{Introduction}

Melanoma is a malignant tumour arising from melanocytes transformation. The benign counterpart of a melanocytic tumour, known as a melanocytic nevus, can be either congenital or commonly acquired, i.e. present at birth or arising during the first decades of life respectively $[1,2]$. The distinction between these two benign entities is classically based on clinical history and presentation. Congenital nevi tend to be more verrucous and hairier. They also show some specific histopathological features such as the presence of nevus cells in the twothirds of the lower dermis, invasion of adnexal structures, perivascular localisation and dispersion between collagen bundles. Congenital nevi are classically divided into two groups, with a distinct risk of progression to malignant melanoma: increased risk for large and giant nevi whereas the malignant progression of smaller lesion seems to be lower [3-6].

Melanocytic tumours are frequently associated with an early driver somatic mutation of the Mitogen-activated protein kinase (MAPK) pathway, mainly on BRAF V600 and NRAS Q61 hotspots. NRAS mutations appeared to be more recurrent in large congenital nevi than in smaller congenital or common nevi. Massively parallel sequencing (MPS) and RNA sequencing identified distinct molecular subtypes of cutaneous melanoma and contributed to a better understanding of the progression from benign to malignant and aggressive melanocytic lesions [7-10]. During the evolution of primary melanoma somatic alterations sequentially induce MAPK pathway activation, upregulation of telomerase by TERT promoter mutation, modulation of epigenetic regulators, override of cell cycle checkpoints, disruption of the p53 pathway, and activation of the PI3K pathway [9].

Here we compared the mutational landscape of congenital and common nevi and explored whether there was a different mutational pattern acquired during progression to melanoma between these two groups.

\section{Methods}

\section{Patients selection}

We retrospectively selected a cohort of 42 patients presenting with a melanoma arising from congenital or common nevus and treated in the oncodermatology unit of the Hospices Civils de Lyon (France)

*Correspondence to: Jonathan Lopez, Biochemistry and Molecular Biology Department, Hospices Civils de Lyon, Centre de Biologie Sud, Centre Hospitalier Lyon Sud, 165 Chemin du Grand Revoyet, 69310 Pierre-Bénite, France, E-mail: jonathan.lopez@chu-lyon.fr

Key words: BRAF, congenital melanoma, nevus transformation, TERT

Received: May 07, 2019; Accepted: June 06, 2019; Published: June 10, 2019 
between 2009 and 2013. From the 637 malignant melanomas arising from nevus initially found in our database over this period, we first selected 114 cases with a Breslow index higher than $0.7 \mathrm{~mm}(17.9 \%)$. Histological and immunohistochemical diagnoses of nevi and related malignant melanomas were then reviewed independently by two expert pathologists $(\mathrm{BB}, \mathrm{OH})$. Based on clinical data and histological criteria, a total of $21(18.4 \%)$ malignant melanomas arising on a nevus with a "congenital nevus pattern" (Cg) were selected $[2,3]$. The control group was composed of 21 malignant melanomas arising on "common nevus pattern" $(\mathrm{Cm})$ matched according to age and sex. Samples were collected for standard care and stored by Hospices Civils de Lyon biobank after obtaining informed consent. We collected size, location, micrometric Breslow index, histopathological subtype and age at diagnosis from medical charts.

\section{Mutational profiling}

Sequencing was performed on paraffin-embedded formalin-fixed (FFPE) specimens after laser microdissection of the nevus and the malignant melanoma compartments on a Leica DM6000B instrument (Leica Microsystems, Germany) (Figure S1). An average of 1,009,480 $\mu \mathrm{m}^{2}$ of tumour cells and $267,061 \mu \mathrm{m}^{2}$ of nevus cells were collected for each case. DNA was extracted from pooled multiple microdissections using QIAamp DNA micro kit (Qiagen, France) and quantified on a Qubit instrument (Life Technologies, UK). BRAF exon 15 and NRAS exon 3 were amplified using in-house primers and sequenced on an $A B I$ PRISM 3730xl instrument (Applied Biosystems, USA). TERT promoter region was amplified using an in-house nested PCR and amplicons were sequenced as described above. We performed Massively Parallel Sequencing of an in-house AmpliSeq panel of targetable cancer genes (Table S1) on micro-dissected melanoma on an Ion Torrent PGM instrument (Thermo Fischer, UK). Of note CDKN2A, ARID2/ARID1A and TP53 genes frequently mutated in melanoma were not included in this panel [9].

\section{Results}

\section{Clinical and histopathological presentation of melanoma arising from congenital and common nevi}

We analysed a total of 42 cases in this study, 21 in each group (see Methods section). All nevi were from small or medium size. There were no large or giant nevi in our cohort, because of the rarity of these lesions. Both groups had similar micrometric Breslow thickness index, AJCC staging, clinical size, male to female ratio and age at the diagnosis of melanoma (Table 1). We didn't observe difference in the location of the melanoma between congenital and common groups. Regarding prognosis, PFS (progression-free survival) was 31.3 months in the congenital group versus 29.1 months in the common melanoma group $(\mathrm{p}=\mathrm{ns})$. Similarly, there was no significant difference in OS (overall survival) between these two groups (34.6 vs 35.1 months).

Thus, in our cohort melanoma arising from nevi of small/ medium congenital and common type share identical clinical and histopathological features and a similar prognosis.

\section{Patterns of BRAF, NRAS and TERT hotspot mutations}

We successfully obtained BRAF and NRAS status for 34 nevi (81\%, 34/42) and 38 malignant melanomas (90.5\%, 38/42) (Table S2). Failure was mainly due to an insufficient DNA quality, highlighting the difficulties to analyse old FFPE samples retrospectively.
Table 1. Clinical and histological characteristics of melanomas

\begin{tabular}{|c|c|c|c|}
\hline & $\begin{array}{l}\text { Congenital nevi } \\
\text { group }\end{array}$ & $\begin{array}{l}\text { Common nevi } \\
\text { group }\end{array}$ & \multirow[t]{2}{*}{ p-value } \\
\hline & $(\mathrm{n}=21)$ & $(\mathrm{n}=21)$ & \\
\hline Age (year) & $41[26-79]$ & $50[27-80]$ & $\mathrm{ns}^{1}$ \\
\hline Sex ratio $(\mathrm{M} / \mathrm{F})$ & $11 / 10$ & $13 / 8$ & $\mathrm{~ns}^{2}$ \\
\hline Clinical size $(\mathrm{mm})$ & $13.5[4-27]$ & $11[4-20]$ & $\mathrm{ns}^{1}$ \\
\hline Breslow index $(\mathrm{mm})$ & $2.06[0.9-5.5]$ & $1.88[0.8-5]$ & $\mathrm{ns}^{1}$ \\
\hline \multicolumn{4}{|l|}{ AJCC staging } \\
\hline 1 & $14(66.7)$ & $16(76.1)$ & \multirow{4}{*}{$\mathrm{ns}^{3}$} \\
\hline 2 & $3(14.3)$ & $1(4.8)$ & \\
\hline 3 & $2(9.5)$ & $1(4.8)$ & \\
\hline 4 & $2(9.5)$ & $3(14.3)$ & \\
\hline \multicolumn{4}{|l|}{ Location } \\
\hline Trunk & $10(47.6)$ & $11(52.4)$ & \multirow{4}{*}{$\mathrm{ns}^{3}$} \\
\hline Limb & $7(33.3)$ & $10(47.6)$ & \\
\hline Acral & $1(4.8)$ & $0(0.0)$ & \\
\hline Face & $3(14.3)$ & $0(0.0)$ & \\
\hline \multicolumn{4}{|l|}{ Survival } \\
\hline PFS (months) & $31.3 \pm 24.0$ & $29.1 \pm 18.7$ & $\mathrm{~ns}^{4}$ \\
\hline OS (months) & $34.6 \pm 20.7$ & $35.1 \pm 14.3$ & $\mathrm{~ns}^{4}$ \\
\hline
\end{tabular}

ns: non-significant; ${ }^{1}$ : paired Student t-test; ${ }^{2}$ : MacNemar test ${ }^{3}:$ Chi-2 test; PFS: progression free survival; OS: overall survival; ${ }^{4} \mathrm{OS}$ and PFS are presented as average survival \pm standard deviation, Age, Clinical size and Breslow index as median [range], AJCC staging and location as $\mathrm{n}(\%)$

BRAF V600E mutation was identified respectively in $70.6 \%(12 / 17)$ of congenital nevi and $64.7 \%(11 / 17)$ of common nevi $(\mathrm{p}=\mathrm{ns})$. The frequency of NRAS Q61 mutation in nevi was $28.6 \%(4 / 14)$ in the congenital group versus $25.0 \%(4 / 16)$ in the common group ( $\mathrm{p}=\mathrm{ns})$. Three nevi ( 2 in the Cg group and 1 in the Cm group) harboured both NRAS and BRAF mutations. Double BRAF/NRAS negative nevi were rare: only 2 in the $\mathrm{Cg}$ group and 3 in the $\mathrm{Cm}$ group.

TERT-promoter sequencing found recurrent C250T or C228T mutation in both melanomas arising from congenital nevi $(5 / 10,50 \%)$ or common nevi $(6 / 9,66.7 \%)$. Importantly none of the paired nevi was mutated for TERT (Table S2). We didn't observe any statistically significant difference of TERT mutation frequency between common and congenital groups. Interestingly we observed a trend towards an increase in TERT mutation rate during tumour progression to metastatic melanoma.

Altogether these results demonstrate that melanoma arising from congenital and common nevi harbour BRAF, NRAS and TERT mutations at a similar rate. They also emphasise a potentially key role of telomerase activation during progression.

\section{Massively parallel sequencing of melanoma}

To get further insights into the dynamic molecular landscape of malignant melanoma arising from common or small congenital nevi, we completed this initial mutational screen by sequencing a pan-cancer MPS panel (see Methods section). We obtained results for 17 patients (Figure 1 and Table S3). Failure was due to the low number of intact DNA in micro-dissected tissue specimen. We were not able to perform MPS on the paired micro-dissected nevi due to a lack of DNA material.

When BRAF or NRAS was mutated in the nevus, the corresponding melanoma also harboured the same mutation. $76.5 \%$ of melanomas harboured additional mutation compared to their related nevi (13/17) with an average of 3 mutations per tumour (52/17). Overall rate of mutations leading to activation of the RAS/RAF/MAPK pathway was 


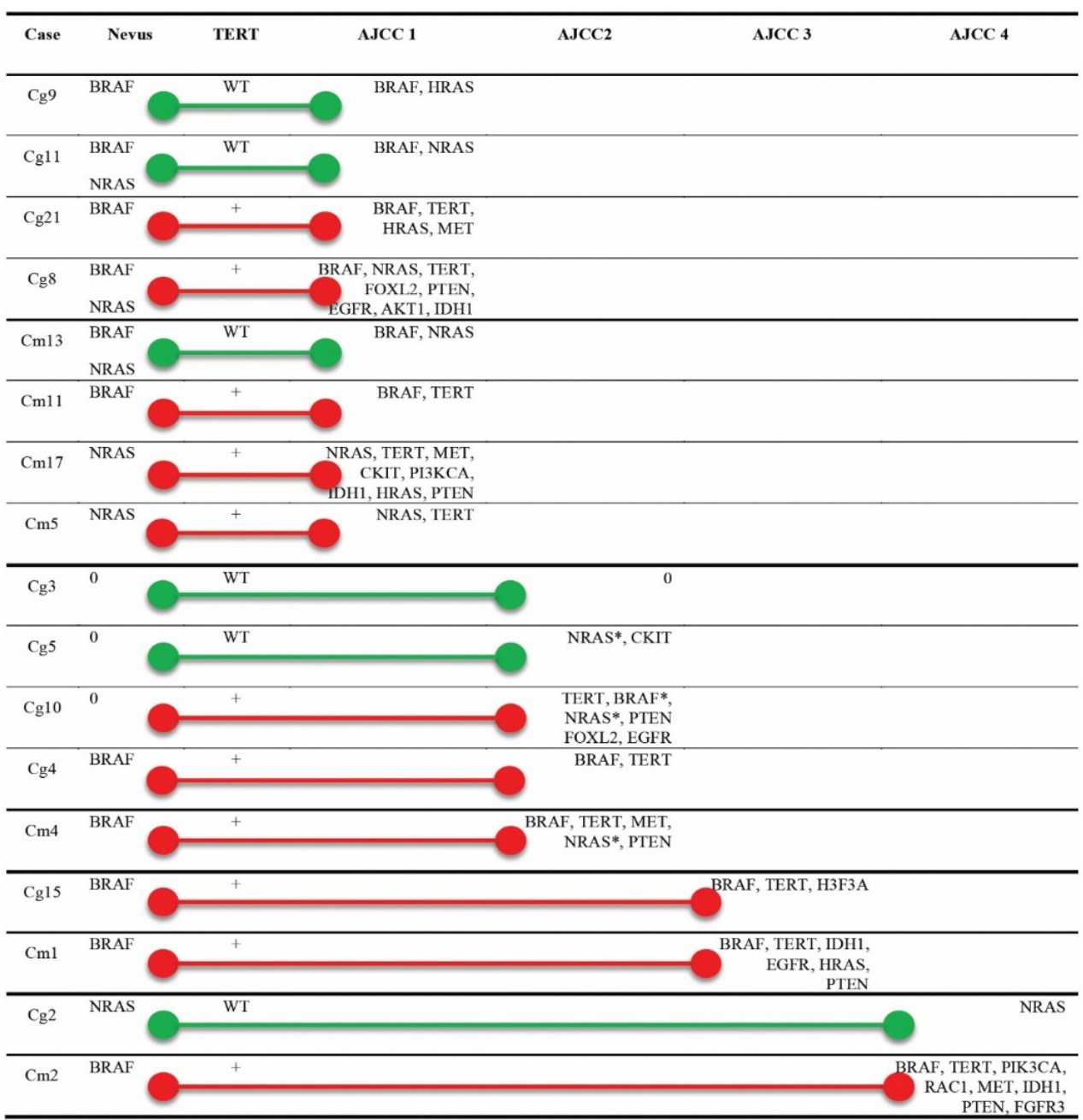

Figure 1. Genomic evolution of melanomas arising from nevi. Cg, congenital group; Cm, common group. AJCC1 to AJCC4, AJCC Melanoma of the skin staging. Green bars: TERT wild type (WT); Red bars: TERT mutated (+). 0, no mutation identified; *, non-canonical BRAF/NRAS mutation

94.1\% (16/17), the remaining tumour (Cg3) being wild type for all the hotspots tested. Other additional mutations identified were affecting the PI3K pathway (loss of PTEN, activation of AKT1 or PIK3CA), the tyrosine kinase receptors signalling (CKIT, EGFR, FGFR3, c-MET) and epigenetic regulators (IDH1, H3F3A). We also observed pathogenic mutations in RAC1 and FOXL2 genes, RAC1 being mutated only in BRAF-mutated metastatic melanomas. An accumulation of mutational events was observed during melanoma progression, from 2 in the AJCC1 group up to 5 in the AJCC4 group. We observed similar patterns of molecular alterations in both small/medium congenital and common groups.

\section{Discussion}

Malignant melanoma arises from nevus in a minority of cases. This retrospective study was designed to compare clinical, histological and mutational profiles of small congenital and common nevi and accumulation of genomic alterations during progression to malignant melanoma.

In our study, nearly all melanocytic nevi (81\%) harboured somatic BRAF/NRAS mutations leading to the activation of the MAPK pathway. As previously reported, the activation of this pathway is sufficient to induce nevi formation $[11,12]$. Here we report that both small/medium congenital and common nevi groups share the same recurrent BRAF/
NRAS driver mutations at similar rates, BRAF V600E being the most prevalent. However, some studies previously reported NRAS Q61 as the sole recurrent mutation in large and giant congenital melanocytic nevi [12]. This discrepancy may be explained by a different clinical size of the lesions in our cohort, BRAF V600E mutation being more frequent in smaller congenital nevi $[13,14]$. In large congenital nevi, progenitor cells with stem cell properties harbouring a somatic NRAS Q61 mutation are maintaining nevi size [15]. BRAF V600 mutation might play a similar role in small or medium-sized nevi, congenital or not. This may explain why both groups present the same malignant risk of progression, lower than the one described in larger tumours $[5,6]$.

Melanomas arising from those small/medium and common nevi harboured some additional mutations when compared to nevi. These alterations were affecting major other oncogenic pathways such as the PI3K pathway, receptor tyrosine kinases signalling, escape to senescence or epigenetic control. Importantly the average number of mutations observed was associated with invasiveness, metastatic melanoma being the most mutated. In line with previous reports, there was an accumulation of mutations with UV-signature, implying that ultraviolet radiation is the dominant mutagen in melanoma, even if there are no sun-exposed signs on histology specimen or in young patients $[9,16,17]$. As for nevi, when comparing mutational profiles of melanoma from congenital and common groups, no clear difference could be made. 
Interestingly, no PIK3CA/AKT1 mutations were found in the NRAS-mutated melanoma whereas half of the BRAF-mutated melanomas also harboured additional activating mutations of the PI3K pathway. This difference may be because NRAS is an activator of both MAPK and PI3K/AKT pathway whereas BRAF activates only MAPK pathway. Similarly, RAC1 mutations were present only in BRAFmutated metastatic melanomas. This pathway has been associated with higher proliferation and resistance to BRAF inhibitors [18]. These additional mutations in BRAF-mutated patients may explain escape to anti-BRAF targeted therapy and emphasise the potential benefit of combined treatment.

We observed a recurrent activation of telomerase by TERT promoter mutation in melanomas but not in nevi, pointing out to a key "second hit" role of TERT in the malignant transformation process. We did observe an increase in TERT mutation rate from AJCC1 lesions to metastatic melanoma. Bastian et al. previously characterised this alteration as an early event in melanoma progression in socalled "dysplastic nevi", an atypical entity of unknown prognosis and management [19]. In such lesions, they found some other mutations frequently reported in melanoma such as CDKN2A inactivation, activation of MAPK or PI3K, suggesting that some of these cases might be malignant melanomas [9].

\section{Conclusion}

To conclude, here we report that melanoma arising from small/ medium congenital and common nevi are sharing a similar mutational landscape. BRAF V600E is the main driver in these nevi. Activation of telomerase by mutating TERT promoter may play an essential role during progression to melanoma as a "second hit".

\section{Conflicts of interest}

None declared

\section{Author contribution statement}

$\mathrm{OH}, \mathrm{GT}, \mathrm{FD}, \mathrm{BB}, \mathrm{LD}$ and $\mathrm{PPB}$ performed the research; SD and JL designed the research study; $\mathrm{OH}, \mathrm{BB}, \mathrm{SD}$ and $\mathrm{JL}$ analysed the data; $\mathrm{OH}$, JC, LT, SD and JL wrote the paper.

\section{References}

1. Krengel S (2005) Nevogenesis--new thoughts regarding a classical problem. Am J Dermatopathol 27: 456-465. [Crossref]

2. Alikhan A, Ibrahimi OA, Eisen DB (2012) Congenital melanocytic nevi: where are we now? Part I. Clinical presentation, epidemiology, pathogenesis, histology, malignant transformation, and neurocutaneous melanosis. J Am Acad Dermatol 67: 495e1-17.
3. Mark GJ, Mihm MC, Liteplo MG, Reed RJ, Clark WH (1973) Congenital melanocytic nevi of the small and garment type. Clinical, histologic, and ultrastructural studies. Hum Pathol 4: 395-418.

4. Bittencourt FV, Marghoob AA, Kopf AW, Koenig KL, Bart RS (2000) Large congenital melanocytic nevi and the risk for development of malignant melanoma and neurocutaneous melanocytosis. Pediatrics 106: 736-741.

5. Rhodes AR, Melski JW (1982) Small congenital nevocellular nevi and the risk of cutaneous melanoma. J Pediatr 100: 219-224. [Crossref]

6. Swerdlow AJ, English JS, Qiao Z (1995) The risk of melanoma in patients with congenital nevi: a cohort study. J Am Acad Dermatol 32: 595-599. [Crossref]

7. Hodis E, Watson IR, Kryukov GV (2012) A landscape of driver mutations in melanoma Cell 150: 251-263.

8. Cancer Genome Atlas Network (2015) Genomic classification of cutaneous melanoma Cell 161:1681-1696.

9. Shain AH, Yeh I, Kovalyshyn I (2015) The genetic evolution of melanoma from precursor lesions. N Engl J Med 373: 1926-1936.

10. Shain AH, Joseph NM, Yu R (2018) Genomic and transcriptomic analysis reveals incremental disruption of key signaling pathways during melanoma evolution. Cancer Cell 34: 45-55.

11. Pollock PM, Harper UL, Hansen KS, Yudt LM, Stark M, et al. (2003) High frequency of BRAF mutations in nevi. Nat Genet 33: 19-20.

12. Charbel C, Fontaine RH, Malouf GG, Picard A, Kadlub N, et al. (2014) NRAS mutation is the sole recurrent somatic mutation in large congenital melanocytic nevi. $J$ Invest Dermatol 134: 1067-1074. [Crossref]

13. Ichii-Nakato N, Takata M, Takayanagi S, Takashima S, Lin J, et al. (2006) High frequency of BRAFV600E mutation in acquired nevi and small congenital nevi, but low frequency of mutation in medium-sized congenital nevi. J Invest Dermatol 126 : 2111-2118. [Crossref]

14. Papp T, Schipper H, Kumar K, Schiffmann D, Zimmermann R (2005) Mutational analysis of the BRAF gene in human congenital and dysplastic melanocytic naevi. Melanoma Res 15: 401-407. [Crossref]

15. Guégan S, Kadlub N, Picard A (2016) Varying proliferative and clonogenic potential in NRAS-mutated congenital melanocytic nevi according to size. Exp Dermatol 25: 789-796.

16. Bastian BC (2014) The molecular pathology of melanoma: an integrated taxonomy of melanocytic neoplasia. Annu Rev Pathol 9: 239-271.

17. Wei X, Walia V, Lin JC (2011) Exome sequencing identifies GRIN2A as frequently mutated in melanoma. Nat Genet 43: 442-446.

18. Araiza-Olivera D, Feng Y, Semenova G, Prudnikova TY, Rhodes J, et al. (2018) Suppression of RAC1-driven malignant melanoma by group A PAK inhibitor Oncogene 37: 944-952.

19. NIH Consensus conference (1992) Diagnosis and treatment of early melanoma

Copyright: (C2019 Harou O. This is an open-access article distributed under the terms of the Creative Commons Attribution License, which permits unrestricted use, distribution, and reproduction in any medium, provided the original author and source are credited. 